\title{
Dependiendo de la nutrición parenteral para sobrevivir. Falla intestinal crónica por síndrome de intestino corto. Comunicación de un caso y revisión de la literatura
}

\author{
Eduardo Moreira*, Patricia López ${ }^{\dagger}$, Lourdes Silvał ${ }^{\ddagger}$ Estela Olano§
}

\section{Resumen}

Cuando la falla intestinal ocurre luego de una resección intestinal masiva se denomina síndrome de intestino corto. Es una entidad rara, con un espectro clínico que va desde una disfunción leve y reversible hasta una condición grave e incapacitante. Presenta una elevada morbimortalidad, altos costos de atención médica e importante impacto en la calidad de vida. El $50 \%$ de los pacientes con síndrome de intestino corto no se adaptan espontáneamente ni mejoran la absorción por el intestino remanente, por lo que deben recurrir de por vida a la nutrición parenteral. La terapia nutricional especializada y el tratamiento hormonal focalizado en el intestino son pilares en la rehabilitación promoviendo la adaptación intestinal, logrando independencia de la nutrición parenteral y evitando el trasplante intestinal. Actualmente, teduglutide es el tratamiento de referencia en estos pacientes, pero hasta el momento inaccesible en Uruguay por su elevado costo. Presentamos el único caso en nuestro país de un paciente de 23 años portador de falla intestinal crónica por síndrome de intestino corto que depende de la nutrición parenteral desde hace 17 años y presenta complicaciones graves asociadas a esta técnica.

Palabras clave: Síndrome del intestino corto

Nutrición parenteral

Teduglutida

Fallo intestinal

Enfermedad crónica

Key words: Short bowel syndrome

Parenteral nutrition

Teduglutide

Intestinal failure

Chronic disease

\footnotetext{
* Médico Intensivista. UCI del Hospital Maciel. Posgrado en Soporte nutricional especializado. Integrante del Equipo Multidisciplinario de Terapia Nutricional del Hospital Maciel. ASSE. Montevideo, Uruguay.

† Prof. Adj. de Clínica Quirúrgica. Integrante del Equipo Multidisciplinario de Terapia Nutricional del Hospital Maciel. ASSE. Montevideo, Uruguay. ¥ Licenciada en Enfermería. Integrante del Equipo Multidisciplinario de Terapia Nutricional del Hospital Maciel. ASSE. Montevideo, Uruguay. $\S$ Médico Intensivista. Coordinadora del Equipo Multidisciplinario de Terapia Nutricional del Hospital Maciel. ASSE. Montevideo, Uruguay. Jefe de la Unidad de Nutrición Especial del Sanatorio CASMU.

Equipo Multidisciplinario de Terapia Nutricional (EMTN).

Unidad de Nutrición Especial y Falla Intestinal. Hospital Maciel.

Correspondencia: Dr. Eduardo Moreira. 25 de Mayo 174, Montevideo, Uruguay. Correo electrónico: dreduardo.moreira@gmail.com

Se cuenta con el consentimiento informado del paciente para la publicación.

Recibido: 20/4/19

Aprobado: 5/8/19
} 


\section{Introducción}

El síndrome del intestino corto severo es una condición rara definida como un intestino delgado remanente con una longitud en continuidad menor de $200 \mathrm{~cm}$. Se caracteriza por la reducción clínicamente significativa de la superficie intestinal capaz de absorber los nutrientes. Es causa importante de falla intestinal crónica o tipo III, donde los cambios estructurales y funcionales favorecen la malabsorción, el riesgo de deficiencias severas de micronutrientes y la inhibición del proceso de desarrollo y crecimiento. La incidencia de síndrome de intestino corto es de aproximadamente 2 casos por millón por año y la prevalencia se sitúa en torno a 20 casos por millón ${ }^{(1-4)}$. La falla intestinal crónica se produce cuando fracasa la fase de adaptación del síndrome de intestino corto ${ }^{(1)}$. La posibilidad de reversibilidad de la falla intestinal crónica depende de la anatomía (figura 1), fisiopatología del síndrome de intestino corto y de la adaptación intestinal, pero la mayoría de los pacientes requieren terapia de soporte nutricional a largo plazo, generalmente en forma de nutrición parenteral crónica (NPC) $)^{(1,5)}$. En la tabla 1 se describen fases fisiopatológicas del síndrome de intestino corto. Anatómicamente, los pacientes con mayor riesgo de falla intestinal crónica y dependencia de NPC son aquellos con una yeyunostomía final y menos de $115 \mathrm{~cm}$ de intestino delgado residual funcional; aquellos con anastomosis yeyuno-cólica y menos de $60 \mathrm{~cm}$ de intestino delgado residual (ausencia de válvula ileocecal), y aquellos con anastomosis yeyuno-ileal y con menos de $35 \mathrm{~cm}$ de intestino delgado residual (pero con presencia de válvula ileocecal y colon). Si bien los pacientes sobreviven, las complicaciones asociadas con la NPC y la falla intestinal crónica son múltiples y graves. Estas incluyen las asociadas con el catéter venoso central (CVC) utilizado para administrar la NPC, la enfermedad hepática asociada a NP y falla intestinal crónica (EHNPFI), las alteraciones óseas y metabólicas, así como el impacto en la calidad de vida ${ }^{(6)}$. El tratamiento de soporte del síndrome de intestino corto se caracteriza por modificaciones en la dieta y terapia farmacológica que se basan en la anatomía y fisiología del remanente intestinal. Estos habitualmente no evitan la NPC. En Europa, se estima que la prevalencia de NP domiciliaria (NPD) para falla intestinal crónica oscila entre 5 y 80 por millón de habitantes, con una incidencia que varía entre 7,7 y 15 pacientes con falla intestinal crónica dependiente de NPC/millón de habitantes/año ${ }^{(3,6)}$.

Las opciones quirúrgicas para el síndrome de intestino corto con falla intestinal crónica, incluido el trasplan-

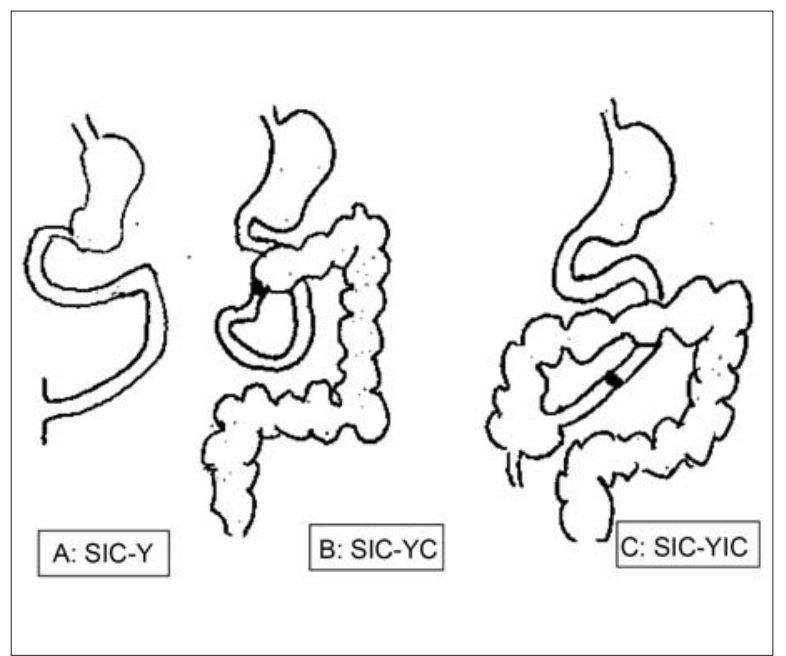

Figura 1. Tipos de intestino corto según la anatomía del intestino remanente.

Grupo A. SIC-Y: síndrome de intestino corto con yeyunostomía o ileostomía final sin colon en continuidad. Grupo B. SIC-YC: síndrome de intestino corto con anastomosis yeyuno cólica sin válvula ileocecal y una parte del colon en continuidad. Grupo C. SIC-YIC: síndrome de intestino corto con anastomosis yeyunoileal con la válvula ileocecal y el colon en continuidad.

Tomada de Ballesteros Pomar M y Vidal Casariego A. Síndrome de intestino corto: definición, causas, adaptación intestinal y sobrecrecimiento bacteriano. Nutr Hosp. 2007;22(Supl. 2):74-85.

te intestinal, están disponibles en algunos centros, pero tienen importantes limitaciones ${ }^{(7-9)}$.

Las nuevas terapias hormonales dirigidas a la adaptación intestinal que se centran en la rehabilitación intestinal mediante la promoción de la hiperadaptación intestinal son prometedoras, en este sentido, el análogo del péptido similar al glucagón -2 (GLP-2) es actualmente el tratamiento de referencia ${ }^{(1)}$.

\section{Caso clínico}

Presentamos el caso de un paciente de sexo masculino de 23 años, estudiante universitario, que a los 6 años (31/8/2002) presentó un infarto intestino mesentérico extenso, requiriendo una enterectomía masiva, quedando como remanente intestinal aproximadamente 10 $\mathrm{cm}$ ( $7 \mathrm{~cm}$ de yeyuno y $3 \mathrm{~cm}$ de íleon con válvula ileocecal preservada). Se realizó el diagnóstico anatomopatológico de panarteritis nodosa, y se completó el tratamiento con glucocorticoides, ciclofosfamida y gammaglobulina.

Con diagnóstico de síndrome de intestino corto anatómico (con reducción significativa de la masa enterocitaria funcionante), requirió internación prolongada en 
Tabla 1. Fases fisiopatológicas del síndrome de intestino corto.

\begin{tabular}{|c|c|c|}
\hline Fase & Duración & Consideraciones clínicas y características fisiopatológicas \\
\hline Fase aguda & Tres a cuatro semanas & $\begin{array}{l}\text { Desórdenes metabólicos y pérdidas intestinales significativas, así como hipersecreción } \\
\text { gástrica debido a la falta de hormonas inhibitorias que normalmente se liberan del íleon } \\
\text { terminal. Requiere una estrecha vigilancia en el entorno hospitalario para evitar secuelas } \\
\text { como la deshidratación con insuficiencia renal aguda, alteraciones ácido-base y } \\
\text { deficiencias de electrolitos. }\end{array}$ \\
\hline $\begin{array}{l}\text { Fase de adaptación. } \\
\text { (Adaptación } \\
\text { espontánea) }\end{array}$ & Uno a dos años & $\begin{array}{l}\text { Cambios adaptativos del intestino delgado remanente para aumentar el área } \\
\text { disponible del intestino para la absorción de nutrientes, disminuir el tránsito } \\
\text { intestinal para maximizar el tiempo disponible para la absorción y la hiperfagia } \\
\text { adaptativa. Estos procesos son promovidos por la presencia de nutrientes en el } \\
\text { intestino, las secreciones pancreáticas y biliares y las hormonas liberadas por el } \\
\text { íleon y el colon. }\end{array}$ \\
\hline $\begin{array}{l}\text { Fase de } \\
\text { mantenimiento. } \\
\text { (Adaptación inducida e } \\
\text { hiperadaptación) }\end{array}$ & Indefinida & $\begin{array}{l}\text { Esta fase se maneja con terapia nutricional (NPC), tratamientos farmacológicos (agentes } \\
\text { antisecretores y antimotilidad) y tratamiento hormonal (somatropina y teduglutide) que se } \\
\text { centra en la rehabilitación intestinal mediante la promoción de la hiperadaptación } \\
\text { intestinal. Eventualmente tratamiento quirúrgico de rehabilitación autóloga, trasplante } \\
\text { intestinal o multivisceral. }\end{array}$ \\
\hline
\end{tabular}

terapia intensiva y múltiples reintervenciones quirúrgicas por peritonitis secundaria hasta la reconstrucción del tránsito digestivo. Luego de ésta, presentó como complicación malabsorción intestinal (alta tasa de diarrea persistente y alteraciones en el crecimiento ponderal), a pesar de la implementación de medidas convencionales de tratamiento (cambios en la dieta oral y enteral, fármacos antisecretores y antimotilidad). Con planteo de falla intestinal tipo II (fase aguda del síndrome de intestino corto), a los 18 días de la primera intervención se colocó catéter venoso central (CVC) para nutrición parenteral total (NPT), se mantuvo nutrición enteral trófica.

Recibió NPC de forma ininterrumpida hasta la actualidad. A los tres años de evolución logró reducción progresiva de las horas diarias de NP y en 2006 se logró el primer día por semana sin NP; evolucionando en los meses siguientes a una falla intestinal tipo III (crónica con dependencia de la NP por síndrome de intestino corto).

Como complicaciones vinculadas a su enfermedad de base y asociadas con la NPC y la falla intestinal tipo III, presentó: a menos de un año del evento (2003) una colecistitis alitiásica, siendo colecistectomizado en esa oportunidad. En 2003, instaló convulsiones tónico-clónicas generalizadas con compromiso encefálico severo que revirtió completamente con ciclofosfamida, gammaglobulina y metilprednisolona. En 2004, presentó hemorragia digestiva alta secundaria a gastritis erosiva por estrés con compromiso hematimétrico y hemodinámico. Entre 2007 y 2009 desarrolló nefrocalcinosis, requiriendo litotricia extracorpórea. Desde los primeros meses, luego del inicio de la NP, desarrolló elementos de enfer- medad hepática asociada con NPC y falla intestinal (EHNPFI), entidad que mantiene hasta la actualidad. Presenta punciones biopsias de hígado que confirman el diagnóstico anatomopatológico de EHNPFI, informándose el 7/7/2015 y el 23/2/2017: cambios inflamatorios portales y lobulillares, esteatosis macrovacuolar leve, focos de balonización hepatocitaria y fibrosis portal moderada (METAVIR 2). Se realizaron ajustes en la composición de la NPC y desde 2012 recibe emulsiones lipídicas de alternativa.

Entre 2010 y 2013, el paciente recibió tratamiento con somatotropina. Actualmente continúa en seguimiento por nefrólogo debido a la presencia de hiperoxaluria.

Desde 2017, recibe NPC domiciliaria nocturna con una fórmula nutricionalmente completa con emulsiones lipídicas de alternativa ahorradoras de lípidos omega 6 , ricas en omega 3 , oligoelementos y vitaminas; se administra en forma cíclica, en infusión de 12 horas diarias, cuatro veces a la semana, por CVC de larga duración. El aporte nutricional semanal actual por vía parenteral es de 5.800 a $6.000 \mathrm{kcal}$, la dieta oral hipercalórica tolerada es mínima, su peso y talla actuales son $58 \mathrm{k}$ y $176 \mathrm{~cm}$. A pesar del tratamiento recibido y del soporte nutricional prolongado con NP, no ha logrado autonomía intestinal e independencia de la NPC, siendo actualmente una terapia de soporte vital (figura 2).

Durante toda su infancia y adolescencia requirió seguimiento estricto por profesionales especializados en nutrición clínica tanto nacionales (de pediatría y adultos) como internacionales; el tratamiento generó impor- 


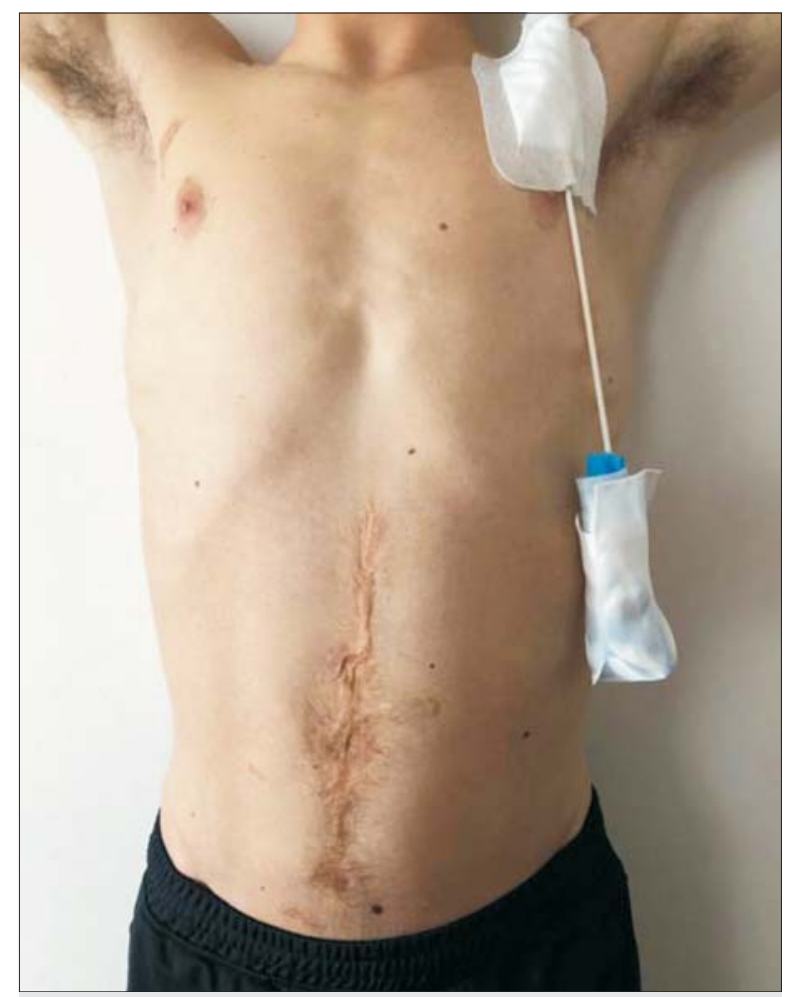

Figura 2. Catéter de larga duración para administración de nutrición parenteral crónica.

tantes limitaciones en múltiples actividades, con impacto en su calidad de vida.

\section{Discusión y comentarios}

La longitud normal del intestino delgado en un adulto, a partir de la flexión duodenoyeyunal, es de 275 a 850 centímetros, a través de los cuales pasan diariamente aproximadamente 9 litros de fluidos (fluidos orales, saliva, secreciones gástricas, biliares y pancreáticas). Entre el $70 \%$ y $80 \%$ de ese líquido se absorbe en el intestino delgado y 2 litros en el intestino grueso. El intestino grueso y sano absorbe aproximadamente 150 kilocalorías por día, pero puede absorber hasta 1.000 kilocalorías por día en aquellos pacientes con malabsorción. La absorción de la mayoría de los nutrientes ocurre en los primeros 100 centímetros del yeyuno, sin embargo, la vitamina B12 y las sales biliares se absorben en los últimos 100 centímetros del íleon; mientras que el magnesio se absorbe en el íleon terminal y el colon proximal y la absorción de agua y sodio ocurre en todo el intestino ${ }^{(10,11)}$. La resección yeyunal limitada suele ser mejor tolerada, ya que la absorción de sales biliares y vitamina B 12 se mantiene normal. La resección ileal se tolera peor, dadas las consecuencias de la pérdida de sales biliares y la limitada capacidad del yeyuno para la hiperplasia adaptativa ${ }^{(10-12)}$. El síndrome de intestino
Tabla 2. Causas más frecuentes del síndrome del intestino corto en adultos.

- Complicaciones de la cirugía abdominal.

- Ocurren más frecuentemente en procedimientos laparoscópicos vs abiertos

- Cirugía bariátrica (vólvulo)

- Enteropatía por radiación o resección tumoral

- Infarto intestino mesentérico (todas las causas)

- Enfermedad inflamatoria intestinal (enfermedad de Crohn)

- Otras causas

corto en adultos se define con menos de 180 a 200 centímetros de intestino delgado remanente, lo que lleva a la necesidad de reposición nutricional y de fluidos ${ }^{(6,11)}$. Alrededor del 75\% de los casos de síndrome de intestino corto se desarrollan después de una resección única y masiva del intestino, como es el caso que presentamos; mientras que el $25 \%$ restante ocurre después de múltiples resecciones ${ }^{(6,11)}$. En la tabla 2 se muestran las causas más frecuentes del síndrome de intestino corto en adultos.

Dos tercios de los pacientes que desarrollan este síndrome sobreviven a su hospitalización inicial, y un número similar sobrevive al primer año luego de desarrollar esta rara entidad. La edad y la enfermedad subyacente muchas veces determinan el resultado a largo plazo de un paciente con síndrome de intestino corto y falla intestinal crónica ${ }^{(6,11)}$. La consecuencia del síndrome de intestino corto es la malabsorción, y este es el principal mecanismo fisiopatológico de la falla intestinal secundaria, debido a la pérdida de la superficie de absorción y al tránsito intestinal acelerado; sin embargo, son múltiples los factores que determinan la gravedad de la malabsorción en el síndrome de intestino corto ${ }^{(6,11)}$. Cuanto mayor es la longitud del intestino delgado que se extrae o se vuelve no funcional, mayor es el área de superficie de absorción perdida. Los criterios fisiopatológicos también pueden subdividirse en categorías donde el colon está en continuidad y aquellos donde no lo está. Estos subgrupos se diferencian en tres características claves: absorción intestinal de agua y sodio, secreción de hormonas gastrointestinales y absorción de energía a partir de ácidos grasos de cadena corta $(\mathrm{AgCC})$ producidos por el microbiota del colon. Los pacientes sin colon en continuidad a menudo pierden más líquido y sodio que los ingeridos (secretores netos), mientras que al igual que nuestro paciente, los que preservan el colon por lo general tienen suficiente in- 
testino distal para permitir el equilibrio hidroelectrolítico (absorbentes netos) y favorecer la adaptación ${ }^{(6,11)}$. Sin embargo, en algunos pacientes la falla intestinal crónica es irreversible y requieren NPC domiciliaria para mantenerse con vida o un trasplante intestinal o multivisceral (hepato-intestinal) si en la evolución aparecen complicaciones potencialmente mortales que surgen como resultado directo de la falla intestinal crónica o secundariamente a la NPC (tabla 3).

Las principales complicaciones de la malabsorción son la desnutrición, la pérdida de peso, la esteatorrea, la diarrea y las deficiencias de vitaminas. Otras complicaciones incluyen nefrolitiasis debida a hiperoxaluria, colelitiasis, hipersecreción gástrica transitoria, crecimiento excesivo de bacterias, deshidratación, hiponatremia, hipopotasemia, insuficiencia renal, colestasis secundaria a enfermedad hepática asociada a falla intestinal crónica y acidosis d-láctica ${ }^{(6,11)}$. En adultos y niños mayores la esteatosis es más frecuente, mientras que en los niños la colestasis y la progresión rápida a fibrosis hepática es la evolución más común ${ }^{(6,11)}$. Hasta el $60 \%$ de las muertes en pacientes con NP domiciliaria son secundarias a EHNPFI $^{(6,11)}$. Nuestro paciente exhibe hallazgos histopatológicos compatibles que incluyen colestasis, esteatosis, esteatohepatitis, ductopenia y fibrosis perivenular y portal.

La falla intestinal crónica es una enfermedad debilitante con un efecto negativo importante en la vida del paciente y su familia ${ }^{(16)}$; es una condición médica compleja que se asocia con altos costos, sobre todo vinculados a la NPD a largo plazo, estos se ven agravados por las admisiones frecuentes secundarias a las complicaciones asociadas. Se desconoce cuáles son las subdivisiones de costos específicos y cómo evolucionan con el tiempo $^{(17)}$.

El manejo inicial del síndrome de intestino corto implica mantener un buen estado nutricional y maximizar la superficie de absorción del intestino remanente, para lo cual es necesario NP posoperatoria temprana, así como nutrición enteral tan pronto como sea posible ${ }^{(6,13)}$. Esto promueve la adaptación intestinal que mejora la absorción de nutrientes con el tiempo. La EHNPFI y la colestasis hepática se tratan manteniendo cierta alimentación oral o enteral, tratando en forma oportuna las complicaciones infecciosas, optimizando la NP en el hogar, disminuyendo la dependencia de las emulsiones de lípidos a base de soja y cambiándolas a emulsiones a base de aceite de pescado. Los pacientes con síndrome de intestino corto (más frecuentemente aquellos con menos de $180 \mathrm{~cm}$ de intestino delgado o una válvula ileocecal ausente) tienen un riesgo aumentado de desarrollar cálculos biliares, habitualmente compuestos de bilirrubinato de calcio; estos pueden prevenirse o revertirse mante-
Tabla 3. Complicaciones asociadas con el síndrome de intestino corto, la falla intestinal tipo III y la nutrición parenteral crónica.

Complicaciones relacionadas con la anatomía del intestino remanente.

- Diarrea por malabsorción

- Desnutrición

- Alteraciones de líquidos y electrolitos

- Deficiencia de micronutrientes

- Deficiencia de ácidos grasos esenciales

- Sobrecrecimiento bacteriano del intestino delgado

- Acidosis D-láctica

- Nefropatía por oxalato

- Insuficiencia renal

- Enfermedad ósea metabólica

- Enfermedad péptica ácida

- Ulceración/estenosis anastomótica

Complicaciones asociadas a la nutrición parenteral crónica (NPC)

- Enfermedad hepática asociada a la FI y NPC (esteatosis, colestasis, fibrosis, cirrosis)

- Complicaciones biliares (cálculos biliares)

Complicaciones relacionadas con los accesos venosos centrales

- Infección

- Oclusión y pérdida de accesos

- Rotura

- Trombosis de la vena central

niendo la nutrición enteral, limitando los períodos de ayuno oral y restringiendo el uso de opioides y anticolinérgicos ${ }^{(10,11,14)}$. Un objetivo primordial cuando se trata a un paciente con falla intestinal crónica por síndrome de intestino corto que requiere NPC es reducir o, cuando sea posible, eliminar su uso. Los medicamentos más utilizados, en particular durante el período de mayor adaptación intestinal, son los antisecretores y los fármacos antimotilidad, necesarios para controlar la hipersecreción gástrica (que se produce de 6 a 12 meses después de la resección de más del 50\% del intestino delgado) y la diarrea de alto débito, existiendo escasa evidencia que respalde su uso ${ }^{(6,15)}$ (tabla 4). Los inhibidores de la bomba de protones en dosis completas pueden reducir las pérdidas de líquido intestinal al disminuir la secreción gástrica. La loperamida y el fosfato de codeína retardan 
Tabla 4. Tratamiento farmacológico utilizado para la inducción de la adaptación intestinal.

\begin{tabular}{|c|c|c|}
\hline $\begin{array}{l}\text { Agentes antisecretores. } \\
\text { Objetivo: control de hipersecreción ácida gástrica }\end{array}$ & Vía de administración & Consideraciones clínicas \\
\hline Antagonistas de los receptores $\mathrm{H} 2$ antihistamina & Vía oral o intravenosa & $\begin{array}{l}\text { - Puede agregarse a la nutrición parenteral } \\
\text { - Disminuye la eficacia con el uso a largo plazo }\end{array}$ \\
\hline Inhibidores de la bomba de protones & Vía oral o intravenosa & $\begin{array}{l}\text { - Requiere un área adecuada de la superficie del } \\
\text { intestino delgado para la absorción oral } \\
\text { - Reevaluar la necesidad a los seis meses } \\
\text { - No se puede agregar a la nutrición parenteral }\end{array}$ \\
\hline Octreotide (análogo de la somatostatina) & Subcutáneo o intravenoso & $\begin{array}{l}\text { - Reservado para diarrea secretora, no osmótica } \\
\text { - Riesgo de hiperglucemia y colelitiasis } \\
\text { - Puede inhibir la adaptación intestinal } \\
\text { - Sobreutilizado en la práctica clínica }\end{array}$ \\
\hline Clonidina & Vía oral o parche & - Riesgo de hipotensión \\
\hline $\begin{array}{l}\text { Agentes antimotilidad. } \\
\text { Objetivo: control de la diarrea }\end{array}$ & Vía de administración & Consideraciones clínicas \\
\hline Loperamida & Vía oral & $\begin{array}{l}\text { - Efectos limitados a nivel central, pero puede } \\
\text { presentarlos a dosis elevadas } \\
\text { - La circulación enterohepática puede limitarse si } \\
\text { existe resección ileal extensa } \\
\text { - Es el agente más utilizado }\end{array}$ \\
\hline Codeína & Vía oral & $\begin{array}{l}\text { - Efecto central } \\
\text { - Debe considerarse cuando falla la terapia con } \\
\text { loperamida } \\
\text { - Puede tener efectos sinérgicos con loperamida } \\
\text { - } 10 \% \text { de metabolizadores rápidos o ultrarrápidos }\end{array}$ \\
\hline $\begin{array}{l}\text { Péptido similar al glucagón-2 (GLP-2). Inductor de la } \\
\text { proliferación epitelial }\end{array}$ & Vía de administración & Consideraciones clínicas \\
\hline Teduglutide & 0,05 mg/k/día subcutáneo & $\begin{array}{l}\text { - Reduce significativamente los requerimientos de NP } \\
\text { o permite independizarse de este soporte } \\
\text { completamente }\end{array}$ \\
\hline
\end{tabular}

el tránsito intestinal de forma segura ${ }^{(6)}$. El octreotide disminuye la secreción y la motilidad gastrointestinal y puede ser útil en algunos pacientes por corto tiempo ${ }^{(13)}$.

Tanto la anatomía del circuito intestinal remanente del caso que presentamos así como su longitud y tiempo de evolución hacen actualmente improbable la rehabilitación intestinal completa con el tratamiento presente, dado que mientras la mitad de los adultos con síndrome de intestino corto y falla intestinal tipo III pueden independizarse completamente de la NPC dentro de los cinco años posteriores al diagnóstico, menos del $6 \%$ aban- donarán la NP utilizando métodos convencionales si no se logra la independencia en los primeros dos años posteriores a la resección ${ }^{(18-20)}$. La reconstrucción gastrointestinal autóloga incluye varias técnicas que manipulan el intestino quirúrgicamente para facilitar el proceso de adaptación intestinal y la restauración de la nutrición enteral. En última instancia, el trasplante intestinal puede servir como la última opción para la cura de la falla intestinal cuando se aplica selectivamente ${ }^{(21)}$.

Dos factores de crecimiento intestinal (somatropina y teduglutide) están disponibles para su uso en pacientes 
con síndrome de intestino corto que no pudieron independizarse de la NPC luego del período de máxima adaptación intestinal $^{(22)}$; nuestro paciente recibió somatotropina. El objetivo de estos tratamientos es reducir la dependencia a largo plazo del soporte parenteral favoreciendo la hiperadaptación del intestino remanente ${ }^{(22,23)}$.

Recientemente, el liraglutide (un análogo de GLP-1) se administró por vía subcutánea una vez al día a ocho pacientes con yeyunostomía terminal en el contexto de un estudio piloto de ocho semanas ${ }^{(24)}$; se demostró reducción del debito de la ostomía, mejoría en la absorción intestinal y producción de diuresis, con escasos y transitorios efectos adversos gastrointestinales leves. Ninguno de estos eventos llevó a la interrupción del tratamiento con liraglutide.

Numerosos trabajos han evaluado recientemente el efecto de otro análogo de GLP-2, en este sentido, un estudio piloto abierto de fase II evaluó por primera vez en 2005 la seguridad y el efecto de teduglutide en pacientes con síndrome de intestino corto ${ }^{(25)}$. El fármaco aumentó significativamente la absorción de masa húmeda absoluta y relativa y disminuyó la masa húmeda fecal y la excreción de energía. También aumentó la altura de las vellosidades, la profundidad de la cripta y el índice mitótico en pacientes con síndrome de intestino corto con yeyunostomía final, cambios que desaparecieron al interrumpir el tratamiento. La aprobación de teduglutide se basó en los resultados de un estudio multicéntrico internacional, en fase III de 24 semanas, doble ciego, controlado con placebo (STEPS-2) ${ }^{(25)}$. Pacientes con falla intestinal por síndrome de intestino corto dependientes de $\mathrm{NP} \geq 3$ veces/semana durante $\geq 12$ meses recibieron $0,05 \mathrm{mg} / \mathrm{kg}$ de teduglutide $(\mathrm{n}=43)$ o placebo $(\mathrm{n}=43)$ una vez al día ${ }^{(26)}$. El número de respondedores fue significativamente mayor en el grupo de teduglutide (27/43, $63 \%)$ respecto al grupo placebo $(13 / 43,30 \%, \mathrm{p}=0,002)$. La reducción media en el volumen de NPC fue de 4,4 \pm 3,8 litros por semana en el grupo de teduglutide vs $2,3 \pm$ 2,7 litros por semana en el grupo placebo $(p<0,001)$. Estos hallazgos fueron confirmados por estudios de seguimiento ${ }^{(27)}$. Según un estudio post hoc que analizó los factores relacionados con la respuesta a teduglutide ${ }^{(28)}$, los efectos del fármaco en el volumen absoluto de NPC fueron significativamente mayores en los pacientes con yeyunostomía/ileostomía terminal (reducción de $919 \pm$ $644 \mathrm{ml} / \mathrm{d}$ ) no solo en comparación con los pacientes que recibieron placebo (reducción de $340 \pm 436 \mathrm{ml} / \mathrm{d} ; \mathrm{p}=$ 0,0112 ), sino también en comparación con los pacientes tratados con teduglutide con $\geq 50 \%$ de colon en continuidad sin estoma, como el caso del paciente que presentamos (reducción de $355 \pm 306 \mathrm{ml} / \mathrm{d} ; \mathrm{p}=0,0066$ ). En pacientes tratados con otras anatomías u otras características de la enfermedad (con enfermedad intestinal in- flamatoria, enfermedades vasculares mesentéricas u otras afecciones) el efecto fue intermedio ${ }^{(28)}$. Los eventos adversos informados fueron los mismos en todos los estudios, en su mayoría vinculados al tracto gastrointestinal (náuseas, vómitos, dolor abdominal) o urinario (infecciones) ${ }^{(29-31)}$.

Rahim Naimi y colaboradores publicaron los primeros datos sobre un nuevo análogo de GLP-2, el glepaglutide, que tiene una vida media de aproximadamente 50 horas. Luego de tres semanas de administración subcutánea diaria los niveles de citrulina (marcador para la masa total enterocitaria) aumentaron en los grupos de intervención y se determinó un vaciamiento gástrico más lento. La producción fecal se redujo significativamente en los grupos de dosis de $1 \mathrm{mg}$ y $10 \mathrm{mg}$ diarios de glepagluti$\mathrm{de}^{(32)}$. En la actualidad un estudio controlado con placebo, multicéntrico, fase 3 (NCT03690206; EASE SBS 1), que evalúa las administraciones una y dos veces por semana, está en curso.

\section{Conclusiones}

Hemos presentado el caso de un paciente adulto joven portador de un síndrome de intestino corto con el tracto gastrointestinal reconstruido con anatomía tipo grupo C (anastomosis yeyuno-cólica con válvula ileocecal y el colon en continuidad) que es portador de una falla intestinal tipo III que no ha logrado independencia de la NP, siendo actualmente necesaria la NPC para mantenerse con vida. Ha presentado una evolución excepcional en nuestro medio por el debut, por su patología de base y por su larga evolución, en la que el enfoque multidisciplinario y el empoderamiento familiar siguen siendo necesarios y resultan esenciales para su supervivencia.

En este paciente en particular, con 17 años de NP y complicaciones graves asociadas a esta técnica de soporte vital, es obligatorio cambiar la perspectiva del tratamiento y dirigirlo a evitar la falla hepática y la muerte. En el mejor de los escenarios, evitar el trasplante combinado (hepato intestinal), procedimiento que no se encuentra disponible en nuestro país. Si bien se esperan resultados adicionales de seguridad a largo plazo, los datos disponibles sugieren que el teduglutide podría resultar esencial, parece ser seguro y efectivo, se ha demostrado que recupera la absorción intestinal y reduce significativamente la dependencia de la NPC, logrando incluso la independencia en algunos casos, mejorando en forma relevante la calidad de vida de pacientes con falla intestinal crónica que dependen de la NPC para sobrevivir.

\section{Abstract}

Short bowel syndrome consists of intestinal failure after a massive intestinal resection. It is an unusual condition, 
ranging from a mild and reversible malfunction to a severe condition causing inability. Short bowel syndrome has high morbimortality, high medical costs and a significant impact on quality of life. $50 \%$ of patients with short bowel syndrome do not spontaneously adapt and neither does absorption improve by the remaining intestine, thus they depend on parenteral nutrition for the rest of their lives.

Specialized nutritional therapy and focalized hormone therapy in the intestine are pillars in rehabilitation. They promote intestine adaptation and achieve independence from parenteral nutrition and avoid intestinal transplant. Today, Teduglutide is the reference treatment for these patients, although it is still not available in Uruguay due to its high cost. The study presents a 23 year old patient carrier of chronic intestinal failure due to short bowel syndrome who has depended on parenteral nutrition for 17 years and presents severe complications associated to this technique.

\section{Resumo}

Quando a falência intestinal ocorre depois de uma ressecção intestinal massiva é chamada Síndrome de intestino curto. É uma entidade rara, com um espectro clínico que pode manifestar-se por uma disfunção leve e reversível até um quadro grave e incapacitante. Apresenta alta morbimortalidade, altos custos de atenção médica e grande impacto sobre a qualidade de vida do paciente. $50 \%$ dos pacientes com síndrome de intestino curto não se adaptam espontaneamente nem conseguem uma melhoria da absorção pelo intestino restante sendo obrigados a utilizar nutrição parenteral de forma permanente. A terapia nutricional especializada e o tratamento hormonal focado no intestino são as bases da reabilitação promovendo a adaptação intestinal, diminuindo a dependência da nutrição parenteral e evitando o transplante intestinal. Atualmente o Teduglutide é o tratamento de referência para estes pacientes, porém devido ao seu elevado custo não é acessível aos pacientes no Uruguai. Apresentamos o único caso no país, de um paciente de 23 anos portador de falência intestinal crônica por síndrome de intestino curto que depende de nutrição parenteral há 17 anos e apresenta complicações graves associadas a esta técnica.

\section{Bibliografía}

1. Pironi L, Arends J, Baxter J, Bozzetti F, Peláez RB, Cuerda C, et al. ESPEN endorsed recommendations. Definition and classification of intestinal failure in adults. Clin Nutr 2015; 34:171-80.

2. Jeppesen PB. Short bowel syndrome - characterisation of an orphan condition with many phenotypes. Expert Opin Orphan Drugs 2013; 1:515-25.
3. Wanden-Berghe C, Virgili Casas N, Ramos Boluda E, Cuerda Compés C, Moreno Villares JM, Pereira Cunill JL, et al. Informe del Grupo de Nutrición Artificial Domiciliaria y Ambulatoria (NADYA)-Nutrición parenteral domiciliaria en España, 2016. Nutr Hosp 2017; 34:1497-501.

4. Pironi L, Konrad D, Brandt C, Joly F, Wanten G, Agostini F, et al. Clinical classification of adult patients with chronic intestinal failure due to benign disease: an international multicenter cross-sectional survey. Clin Nutr 2018; 37:72838 .

5. Neelis EG, Olieman JF, Hulst JM, de Koning BA, Wijnen RM5, Rings EH. Promoting intestinal adaptation by nutrition and medication. Best Pract Res Clin Gastroenterol 2016; 30(2):249-61.

6. Pironi L, Arends J, Bozzetti F, Cuerda C, Gillanders L, Jeppesen PB, et al. ESPEN guidelines on chronic intestinal failure in adults. Clin Nutr 2016; 35(2):247-307.

7. Beyer-Berjot L, Joly F, Maggiori L, Corcos O, Bouhnik Y, Bretagnol F, et al. Segmental reversal of the small bowel can end permanent parenteral nutrition dependency: an experience of 38 adults with short bowel syndrome. Ann Surg 2012; 256:739-44.

8. Layec S, Beyer L, Corcos O, Alves A, Dray X, Amiot A, et al. Increased intestinal absorption by segmental reversal of the small bowel in adult patients with short-bowel syndrome: a case-control study. Am J Clin Nutr 2013; 97:100-8.

9. Grant D, Abu-Elmagd K, Mazariegos G, Vianna R, Langnas A, Mangus R, et al. Intestinal transplant registry report: global activity and trends. Am J Transplant 2015; 15:210-9.

10. Semrad C. Aproximación al paciente con diarrea y malabsorción. En: Goldman-Cecil. Tratado de medicina interna. $25^{\mathrm{a}}$ ed. Barcelona: Elsevier, 2017:918-35.

11. Pironi L. Definitions of intestinal failure and the short bowel syndrome. Best Pract Res Clin Gastroenterol 2016; 30(2):173-85. doi: 10.1016/j.bpg.2016.02.011.

12. Tappenden KA. Pathophysiology of short bowel syndrome: considerations of resected and residual anatomy. JPEN J Parenter Enteral Nutr 2014; 38(1 Suppl):14S-22S.

13. Nightingale J, Woodward JM. Guidelines for management of patients with a short bowel. Gut 2006; 55(4 Suppl):iv1-12.

14. Parrish CR, DiBaise JK. Managing the adult patient with short bowel syndrome. Gastroenterol Hepatol (N Y) 2017; 13(10):600-8.

15. Chan LN, DiBaise JK, Parrish CR. Short bowel syndrome in adults-Part 4A. A guide to front line drugs used in the treatment of short bowel syndrome. Practical Gastroenterol 2015; 39(3):28-42.

16. Winkler MF, Smith CE. Clinical, social, and economic impacts of home parenteral nutrition dependence in short bowel syndrome. JPEN J Parenter Enteral Nutr 2014; 38(1 Suppl): 32S-7S.

17. Canovai E, Ceulemans LJ, Peers G, De Pourcq L, Pijpops M, De Hertogh G, et al. Cost analysis of chronic intestinal failure. Clin Nutr 2018; 38(4):1729-36. doi:10.1016/j. clnu.2018.07.032. 
18. Messing B, Crenn P, Beau P, Boutron-Ruault MC, Rambaud JC, Matuchansky C. Long-term survival and parenteral nutrition dependence in adult patients with the short bowel syndrome. Gastroenterology 1999; 117(5):1043-50.

19. Amiot A, Messing B, Corcos O, Panis Y, Joly F. Determinants of home parenteral nutrition dependence and survival of 268 patients with non-malignant short bowel syndrome. Clin Nutr 2013; 32:368-74.

20. Jeppesen PB. The non-surgical treatment of adult patients with short bowel syndrome. J Expert Opin Orphan Drugs 2013; 1:527-38

21. Solar H, Crivelli A, Echevarria C, Ramisch D, Luis Moulin, Fabio Nashman, et al. Autologous gastro-intestinal reconstruction in adult patients with severe intestinal failure. Report of long term outcomes and its correlation with anatomical predicting factors. Report from a single center. Abstracts from the XIV International Small Bowel Transplant Symposium. Transplant 2015; 99(6S-1 Suppl):S107. Abstract $n^{\circ} 655$.

22. Pape UF, Maasberg S, Pascher A. Pharmacological strategies to enhance adaptation in intestinal failure. Curr Opin Organ Transplant 2016; 21:147-52.

23. Gondolesi GE, Pattín F, Nikkoupur H. Management of intestinal failure in middle-income countries, for children and adults. Curr Opin Organ Transplant 2018; 23(2):212-8.

24. Hvistendahl M, Brandt CF, Tribler S, Naimi RM, Hartmann B, Holst JJ, et al. Effect of liraglutide treatment on jejunostomy output in patients with short bowel syndrome: an open-label pilot study. JPEN J Parenter Enteral Nutr 2016; 42(1):112-21

25. Jeppesen PB, Sanguinetti EL, Buchman A, Howard L, Scolapio JS, Ziegler TR, et al. Teduglutide (ALX-0600), a dipeptidyl peptidase IV resistant glucagon-like peptide 2 analogue, improves intestinal function in short bowel syndrome patients. Gut 2005; 54:1224-31.

26. Jeppesen PB, Pertkiewicz M, Messing B, Iyer K, Seidner DL, O'keefe SJ, et al. Teduglutide reduces need for parenteral support among patients with short bowel syndrome with intestinal failure. Gastroenterology 2012; 143:1473-81.e3.

27. Schwartz LK, O'Keefe SJ, Fujioka K, Gabe SM, Lamprecht G, Pape UF, et al. Long-term teduglutide for the treatment of patients with intestinal failure associated with short bowel syndrome. Clin Transl Gastroenterol 2016; $7: \mathrm{e} 142$.

28. Jeppesen PB, Gabe SM, Seidner DL, Lee HM, Olivier C. Factors associated with response to teduglutide in patients with short-bowel syndrome and intestinal failure. Gastroenterology 2018; 154:874-85.

29. Billiauws L, Bataille J, Boehm V, Corcos O, Joly F. Teduglutide for treatment of adult patients with short bowel syndrome. Expert Opin Biol Ther 2017; 17:623-32.

30. Ring LL, Nerup N, Jeppesen PB, Svendsen LB, Achiam MP. Glucagon like peptide-2 and neoplasia; a systematic review. Expert Rev Gastroenterol Hepatol 2018; 12:257-64.

31. Kochar B, Herfarth HH. Teduglutide for the treatment of short bowel syndrome - a safety evaluation. Expert Opin Drug Saf 2018; 17(7):733-9.

32. Naimi RM, Hvistendahl M, Enevoldsen LH, Madsen JL, Fuglsang S, Poulsen SS, et al. Glepaglutide, a novel long-acting glucagon-like peptide-2 analogue, for patients with short bowel syndrome: a randomised phase 2 trial. Lancet Gastroenterol Hepatol 2019; 4(5):354-63. doi:/10.1016/S 2468-1253(19)30077-9.

\section{Contribución de autores}

Todos los autores participaron en igual medida en la elaboración del manuscrito.

Eduardo Moreira, https://orcid.org/0000-0002-2522-3230

Estela Olano, https://orcid.org/0000-0002-8101-8979

Lourdes Silva, https://orcid.org/0000-0002-2259-2313

Patricia López, https://orcid.org/0000-0003-0951-6831 